\title{
Effects of a Six-week Pedometer-based Walking Programme on Physical Activity Level, Body Composition and Antioxidant Level in Breast Cancer Survivors: a Randomized Controlled Trial
}

\author{
A. D. Ezuma \\ University of Nigeria Teaching Hospital
}

S. C. Ibeneme ( $\square$ sam.ibeneme@unn.edu.ng )

University of Nigeria

G. Fortwengel

Hochschule Hannover University of Applied Sciences and Arts

\section{H. Myezwa}

University of the Witwatersrand

\section{A. T. Ajidahun}

University of the Witwatersrand

E. Ezeome

University of Nigeria Teaching Hospital, Ituku/Ozalla

\section{A. Lasebikan}

University of Nigeria Teaching Hospital, Ituku/Ozalla

C Obiekwe

University of Nigeria Teaching Hospital

\section{A. Nnamani}

University of Nigeria Teaching Hospital, Ituku/Ozalla

\section{Okoye}

University of Nigeria Teaching Hospital, Ituku/Ozalla

\section{T.A.O Onodugo}

University of Nigeria Teaching Hospital

\section{Research Article}

Keywords: Breast cancer survivors, pedometer-based physical activity lifestyle promotion, physical activity level, body composition, superoxide dismutase

Posted Date: February 2nd, 2022 
DOI: https://doi.org/10.21203/rs.3.rs-1204264/v1

License: (c) (1) This work is licensed under a Creative Commons Attribution 4.0 International License. Read Full License 


\section{Abstract}

Background: Breast cancer risk is associated with body composition indices especially waist-hip ratio$\geq 0.85$ (which defines central adiposity), body mass index $\geq 30 \mathrm{Kg} / \mathrm{m} 2$ (which defines obesity), percentage body fat mass $-25 \mathrm{Kg} / \mathrm{m} 2$ (which defines general adiposity), and physical inactivity (defined as $<600$ (MET-min/week or $\leq 7999$ step counts/day). Physical inactivity promotes inflammatory microenvironment, oxidative stress and diminish antioxidant (superoxide dismutase) level, similar to adiposity but unlike physical activity. Therefore, a pedometer-based walking programme may improve body composition, physical activity and Superoxide dismutase levels in breast cancer survivors, thus preventing breast cancer recurrence.

Methods: This study is a single-blind randomized controlled trial involving 80 participants from the Oncology Clinic, who were consecutively stratified according to their baseline physical activity level (METmin/week), and step count/day obtained using a Fitbit Charge HR pedometer. The experimental group received an intervention programme to improve physical activity level by setting weekly targets of additional 1500 steps to the baseline value, while the control group did not change their physical activity tasks for the duration of the study. Data were collected with the short form of International Physical Activity Questionnaire at baseline and the $6^{\text {th }}$ week, and weekly from the Fitbit server/data centre, which receives transmitted data from the pedometer customised to each participant. Data were analysed using simple descriptive statistics, multiple regression analysis, and independent t-test at $p<0.05$, one-tailed test.

Results: There was a significant increase in step counts/day $(p=0.0002)$, physical activity level(MET$\mathrm{min} /$ week $)(p=0.006)$, and Superoxide dismutase level $(p=<.0001)$, with a decrease in waist-Hip Ratio $(p=0.002)$, and percentage body fat mass $(p=0.01)$, unlike the body mass index $(p=0.09)$ in the intervention group compared to control.

Conclusion: Physical activity, Superoxide dismutase level and body composition may be improved through a pedometer-based walking programme and possibly the prognosis of breast cancer recurrence.

Trials Registration number: PACTR202001542824141.

\section{Introduction}

Breast cancer is the most common among women and recent estimates showed that there are 2 million new cases and 627000 women died from breast cancer in 2018. Modifiable risk factors associated with breast cancer includes - body composition, diet, exercise, smoking and alcohol consumption. Body composition is a risk factor for breast cancer, especially waist-hip $\geq 0.85$ (which defines central adiposity) [1], body mass index $\geq 30 \mathrm{Kg} / \mathrm{m} 2$ (which defines obesity) [2,3], and percentage body fat mass $\geq 25 \mathrm{Kg} / \mathrm{m}^{2}$ which defines general adiposity) [4]. 
Adiposity promotes inflammatory micro-environment, oxidative stress and diminish antioxidant such as superoxide dismutase level, but responds positively to physical activity. There is evidence that a lifestyle of physical activity may promote antioxidant activity/level, relieve inflammation, boost immune function, and improve body composition [5-7], all of which ameliorate the risk factors for breast cancer. Therefore, a pedometer-based physical activity lifestyle promotion strategy may play a strategic role in realizing the goal of public health campaign for the prevention of breast cancer recurrence, while the pedometer enables the quantification of physical activity in step counts/day that may allow this. Pedometers allow for the quantification of walking steps within daily physical activity log and thus, provides a measurable approach for estimating proportionate health benefits when related to the molecular biomarkers that lie on the path of biological plausibility for breast cancer recurrence/induction. Yet, many studies conducted on breast cancer and physical activity in the African population [8-12] and particularly, Nigerian population [13-18] are descriptive (hence physical activity is not objectively quantified), provided no evidence of causal relationships and none included molecular investigations. These studies highlight the difficulty of determining the level of evidence, in non-molecular studies that may be of clinical relevance. Therefore, if surrogate markers of breast cancer prevention, such as serum level of superoxide dismutase, (as an outcome measure rather than cancer itself), are not calibrated in response to quantifiable indicators of physical activity, such as pedometer step counts/day, it may be difficult to estimate the quantity of physical activity required for breast cancer prevention or its recurrence in public health promotion. The quantification of physical activity is important in Nigeria, where physical inactivity prevalence is $41 \%$, and the females are more likely to be inactive [19]. Consequently, pedometer-based change in physical activity lifestyle may not only help this population to become active but provides measurable data on which basis such a change in physical activity can be related to proportionate molecular responses to guide practice. These gaps in knowledge justified the need for the present study in Nigeria, whose population accounts for $20 \%$ of the population of Africa, and slightly more than half the population of West Africa, yet Nigeria contributed $15 \%$ to the estimated 681,000 new cases of cancer that occurred in Africa in 2008 alone [20, 21].

Recent Global Cancer Incidence, Mortality and Prevalence (GLOBOCAN) estimate indicates that there were 115950 new cases of cancer in 2018 out of which 26310 (22.7\%) were breast cancer cases with cancerrelated deaths reported at 70327 [22]. A significant proportion of the increase in the incidence of breast cancer in Nigeria is due to increasing life expectancy, reduced risk of death from infectious diseases, increasing prevalence of smoking, obesity as well as changing dietary and lifestyle patterns, especially physical inactivity $[20,21]$. Regular physical activity is directly related to physiological and psychological health benefits $[23,24]$. However, most breast cancer survivors experience cancer related fatigue [25] and are unlikely to achieve the minimum requirements of at least 150 minutes a week of moderate-intensity physical activity for health [26]. Thus, a high proportion of breast cancer survivors are sedentary/irregularly active [27, 28]. A sedentary lifestyle has been linked to weight gain [29, 30]. However, weight gain of up to $2-6 \mathrm{~kg}$ which is likely to alter body composition [31-33], is also a common problem among individuals receiving adjuvant chemotherapy (cyclophosphamide, methotrexate, and fluorouracil) for breast cancer [34]. The consequent weight gain/obesity may affect upper and lower extremity muscle 
strength [31], impair walking function and increase physical inactivity. On the contrary, there is evidence that physical exercises at $25 \%, 50 \%$ and $75 \%$ of maximal oxygen consumption $\left(\mathrm{VO}_{2}\right.$ max.) enhanced the activity (lysis per fixed number of mononuclear cells) of macrophages [35], neutrophils, regulating cytokines, natural killer cells and lymphokine-activated killer cells that antagonize cancer cells [36-38]. In essence, a pedometer-based walking programme targeted at improving physical activity level may constitute an important component of a behavioural intervention strategy for breast cancer prevention/management. In this regard, brisk walking has been identified as the form of physical activity most apt to boost physical activity $[39,40]$ and is the most commonly reported mode of physical activity amongst adults in many populations [41, 42]. However, the use of pedometer allows for quantification of walking steps within an individual's daily physical activity log. Importantly, quantifying physical activity provides a measurable approach for monitoring progress and relating the same to the proportionate response of the biological system to lifestyle modifications. Though the efficacy of pedometer-based physical activity lifestyle promotion strategy in achieving improved physical activity level has been demonstrated in adults [42,43], it is not known if it could have the same effects in breast cancer survivors and significantly boost the biomarkers of cancer prevention/recurrence (such as superoxide dismutase) in a Nigeria population. The primary endpoint is physically-active survival. It is defined as the time point from the date of randomization to the date of becoming physically inactive or death due to any cause. This trial further sought to evaluate the improvements in body composition, antioxidant level and overall survival as secondary endpoints. Therefore, this study primarily sought to determine the effects of a 6week pedometer-based walking programme on: i. physical activity level (MET-min/week and), The secondary objectives sought to determine the effects of a 6-week pedometer-based walking programme on: ii. body composition parameters (Body mass index, waist-hip ratio, and percentage body fat mass), and iii. serum level of antioxidants (Superoxide dismutase). There were analyses of the physical activity level and body composition parameters among breast cancer survivors to provide detailed patientspecific data on whether there were changes in the categorisation of breast cancer survivors according to their: iv. physical activity level (MET-min/week), and, v. body composition parameters after a 6-week pedometer-based walking programme. It was hypothesized that a 6-week pedometer-based walking programme will significantly increase physical activity level (MET-min/week), serum antioxidant (superoxide dismutase) level, and improve body composition parameters in breast cancer survivors.

\section{Methods}

\section{Study design:}

This is an assessor-blinded randomized controlled trial that utilized a simple random sampling technique in sample selection. This study was registered with the Pan African Clinical Trials Registry - Trial ID: PACTR202001542824141, (https://pactr.samrc.ac.za/Search.aspx) on the 28/01/2020.

\section{Participants}


To be eligible to participate in this trial, women were: i. Diagnosed with breast cancer, ii. Aged $18-60$ years, iii. Ambulating independently, iv. Not on sedatives at least 4 weeks prior to the study (since it decreases systemic vascular resistance due to inhibition of vasoconstrictor activity of the sympathetic nervous system, decreases cardiac contractility, and decreases preload ${ }^{46}$, v. Had no history of hypertension (because anti-hypertensive drugs influence the neurohumoral cardiovascular system and the concentration of hormones involved in blood pressure regulation 47 , vi. Staging of breast cancer was within stages I - III according to Surveillance, Epidemiology, and End Results (SEER) stage of disease classification, ${ }^{48}$ vii. Never-smokers (since smoking influences the serum level of Superoxide dismutase) ${ }^{49}$, viii. On primary treatment (chemotherapy, radiotherapy surgery) for $\leq 2$ months, and ix. Without a visual loss. Participants were excluded if they had a history of other underlying pathological diseases of metabolic, orthopaedic or neurological nature.

\section{Study setting}

The study site was primarily at the Oncology centre, University of Nigeria Teaching Hospital, Ituku/Ozalla, Enugu, Enugu State, Nigeria. Enugu State, has an estimated population of 3.8 million, and is one of the 36 states of Nigeria.

\section{Intervention}

\section{a. Intervention arm}

The primary intervention comprised of two components delivered over a six-week period.

\section{- Behavioural feedback and goal-setting session}

Physical activity consultations, behavioural feedback and goal-setting sessions were held with the participants to address the barriers to physical activity using the TTM [49], throughout the study duration. The TTM is a common theoretical framework for physical activity consultations [50], which has published guidelines for health professionals to conduct consultations [51, 52] and has been used successfully in intervention studies designed to increase physical activity $[53,54]$. According to the guidelines, the consultations were semi-structured [51, 52], and adopted a guiding style by which the participants made decisions about how to modify and promote their walking behaviour [55] using various strategies. These strategies included: enhancing motivation, overcoming barriers and developing appropriate walking plans which were personalized to the individual as recommended by Ogilvie and colleagues [56]. The sessions also included a reflection on the three mediators of The TTM required for behaviour change [49], namely, i. self-efficacy (confidence in the ability to change), ii. decisional balance (pros and cons of change) and iii. processes of change (strategies and techniques used to change, e.g., social support). Although the sessions were flexible and personalized, The TTM was used to create a standard protocol and steps that were followed during the sessions (Table 1). 
Table 1

Weekly goals of intervention group participants

\section{Time-point Goal}

Week 1 To walk an extra 1,500 steps (from baseline value) on at least 3 days of the week

Week 2 To walk an extra 1,500 steps (from baseline value) on at least 3 days of the week

Week 3 To walk an extra 1,500 steps (from baseline value) on at least 5 days of the week

Week 4 To walk an extra 1,500 steps (from baseline value) on at least 5 days of the week

Week 5 To walk an extra 3,000 steps (from baseline value) on at least 3 days of the week

Weeks 6 To maintain walking levels using the week 5 goal

\section{- The walking programme}

The walking programme lasted for six weeks, and varied as follows: the first 5 weeks consisted of graduated weekly goals with an aim for the increased walking behaviour to be maintained for the remaining one week (at the $6^{\text {th }}$ week). The overall goal of the walking programme was for the participants to increase their mean daily step-count by 3,000 accumulated steps above their baseline value on five days of the week. Three thousand steps is based on the assumption that moderate brisk walking produces 100 steps a minute (1,000 steps per 10 minutes) [57]. Therefore, 3,000 steps would equate to approximately 30 minutes of moderate physical activity, in line with current physical activity recommendations [58]. This program has previously been used in sedentary adults, in the United Kingdom over a shorter time-frame [59]. Goals were retained for one week to enable participants to reinforce their increased levels of walking or to try other strategies to successfully accumulate the additional steps. Participants were advised on the nature of the intensity and duration of the desired increases in walking. Participants were familiarized with the Borg 6-20 scale [60] on which basis they were advised to ensure that the additional walking was a brisk nature that would leave them slightly breathless and hot, but still able to talk (indicated as between 12-14 on this scale). Bouts of at least 10 minutes in duration were recommended for additional walking, although the accumulation of walking during everyday tasks as much as possible was also advocated. The participants in the intervention group collected their pedometers at the end of baseline assessment visit and wore it unsealed until the end of week six of the study.

\section{b. Control arm}

Participants assigned to the control group were asked to maintain their normal physical activity level/walking behaviour between baseline and week six. They were advised to continue their current management program and scheduled for follow-up at the end of week five. The participants in the control group collected their pedometers at week 0 , and wore it sealed in order to gain a record of their daily 
pedometer step counts at baseline. They returned it at the end of week 0 , but were given the pedometers again at the end of week five, and were required to wear it sealed all through week six to gain a record of their step-counts. At the end of week 0 and week 6 , other health outcomes apart from pedometer step counts/day, were also measured among all the participants. Prior to the assessment session, each participant was rested for 30 minutes.

\section{Outcome variables}

The outcome variables of interest that were measured in this study include:

- A. Independent variables

- 1. Physical Activity

The overall daily physical activity was measured using two methods, namely pedometer step counts/day and physical activity expressed as MET-min per week

- Pedometer step counts/day: The primary outcome measure is pedometer step counts/day measured by Fitbit Charge HR (Fitbit International Ireland). This was measured as step counts/day. The cut off points according to Jordan et al [70] are:

- low step count $=\leq 7,999$ step counts/day

- high step count $=\geq 8000$ step counts/day

- Physical activity level (MET-min/week). The cut-off points according to International Physical Activity Questionnaire (IPAQ) criterion score [65] are: -

- high physical activity level=3000 MET-min/week

- moderate physical activity level=600-3000MET-min/week

- low physical activity level = 600MET-min/week

- B. Dependent variables

- 2. Body composition. The cut-off points according to World Health Organisation (WHO) [1] are: -

- Waist-to-hip ratio according to previous studies [1-3] is categorised into:

- normal adiposity for females $=<0.85$;

- abdominal adiposity/obesity $=>0.85$

- Body mass index according to previous studies [1-3] is categorised into:

- normal weight category $=\leq 20 \mathrm{Kg} / \mathrm{m}^{2}-\geq 25 \mathrm{Kg} / \mathrm{m}^{2}$

- overweight $=25 \mathrm{Kg} / \mathrm{m}^{2-}-29 \mathrm{Kg} / \mathrm{m}^{2}$

- Obesity $=\geq 30 \mathrm{Kg} / \mathrm{m}^{2}$

- Percentage body fat mass: Cut-off for percentage body fat mass based on previous studies [71, 72] are: 
- Percentage body fat for the average population of females aged $30-50$ years $=20-30 \%$.

- Overweight $=>30 \%-<32 \%$

- Obesity $=\geq 32 \%$

- 3. Serum level of Superoxide dismutase - normal range for females is from $7.8-17.6 \mathrm{U} / \mathrm{ml}$ [73].

\section{Sample size}

The sample size of this study is based on our primary aim: to determine the efficacy of the pedometerbased physical activity behaviour promotion in influencing physical activity level of breast cancer survivors, of which the primary outcome variable is minutes per week of moderate to vigorous activity. Using the statistical superiority sample size design formula [44], assuming a between group change of 75 minutes per week in moderate to vigorous physical activity (MVPA), standard deviation of change of 120 minutes [45], power of $80 \%$ and $95 \%$ confidence interval, the estimated sample size accounting for $10 \%$ dropout is 44 participants per group $(\mathrm{N}=88)$.

\section{Randomisation, sequence generation and allocation}

Randomisation, sequence generation and allocation of participants were done after their baseline assessment to determine the step count/day and physical activity level on which basis they were stratified, randomised and allocated into groups.

\section{- Baseline assessment of the stratification variable}

At onset, the participants collected an individually-calibrated pedometer from the research centre and wore it sealed during the week zero to gain a record of their baseline step-counts/day. All participants completed a baseline week wearing a pedometer for seven days at all times (except when showering, sleeping or taking part in structured sport or exercise (e.g., athletics, gym workouts, football, dance aerobics, etc.) with instructions not to alter their daily routine. Irregular bouts of structured sport or exercise that could significantly affect an individual's mean step-count was recorded. Each pedometer was individually calibrated consistently with manufacturers guidelines to within $5 \%$ of actual steps walked in a 100-step test. Participants were required to provide at least five days of step counts including at least one weekend day to gain an accurate reflection of physical activity levels. At the end of the baseline week, the participants returned the pedometers to the study centre. During the visit, the baseline The International Physical Activity Questionnaires (IPAQ) scores of each participant was recorded. The participants were subsequently stratified into low (<600MET$\mathrm{min} /$ week), moderate (600-3000MET-min/week) and high levels (3000 MET-min/week) of physical activity on the basis of their total physical activity (MET-min/week) and the frequency of activities. ${ }^{70}$ The participants within each IPAQ score category above were further stratified into two groups according to their step counts/day using baseline low step count of $\leq 7,999$, and high step count of $\geq 8000$. The value of 8,000 step count/day was used as a stratification variable to account for 
individuals with a high baseline step-count. This value was previously used as a baseline descriptor for sedentarism. ${ }^{73}$

From a frame of the breast cancer survivors who met the eligibility criteria, participants were randomized into groups using computer-generated numbers and concealed allocation. Thus, using block allocation, participants from each of the stratum mentioned previously (i.e., low step count of $\leq 7,999$, and high step count of $\geq 8000$ ) were also randomized into the two study groups, namely: -

a. Intervention group - which comprised of participants who were given a pedometer to monitor their step counts/day, and,

b. Control group - which comprised of patients without a pedometer to monitor their step counts/day.

To achieve this, random numbers were generated to allocate the patients into intervention and control groups in blocks (10 numbers equally distributed into intervention and control groups). The blocks were divided into two, namely - low step count of $\leq 7,999$, and high step count of $\geq 8000$, and concealed in an envelope. The number allocation was done by independent research support and was done in such a manner that until the 10 numbers in an envelope were exhausted before a new block of 10 numbers were allocated. This process was continued until the sample was exhausted.

\section{Blinding}

The principal investigator, who was the assessor, was blinded to allocation. A qualified registered physiotherapist was appointed as the research assistant 1 (RA 1) for the study. The research assistant 1 kept the names of the randomised participants in a centrally locked steel-drawer and arranged for the participants to undergo assessment by the assessor without informing the assessor which groups a participant belonged to. The research assistant 1 implemented the intervention programme and did not allow the assessor access to the groups during intervention period.

\section{Data Collection}

\section{Recruitment}

Recruitment strategy involved 6-week publicity campaign about the study involving public health talks for Breast cancer survivors who attended the Oncology Clinic., Flyers were placed in the Physicians consulting rooms in private and public health facilities in Enugu Metropolis, Enugu State, Nigeria. Announcements were made in local religious congregations, interested participants contacted the researcher through email, phone number, and social media handle provided in newsletters. Interested participants were screened for eligibility and recruitment started on 27 July, 2016. The study lasted from 27 July 2016 and 26 July, 2017. The participants were followed-up from 27 July 2017 to 26 January, 2018. Each participant had a documented diagnosis, treatment and history of being followed-up at the Oncology Clinic. The time of recruitment and assessment was the usual routine clinic hours $(8-4 \mathrm{pm})$ at the oncology clinic. 


\section{Procedure.}

The study process involved three stages: obtaining informed consent, assessment of body anthropometry and physical activity profile, and laboratory study. At the onset of the study, written informed consent was obtained from all participants prior to their involvement in the pedometer-based walking intervention trials. All participants were provided with a diary to record their daily physical activity, a pedometer with instructions on how to use it and IPAQ-SF questionnaire for baseline and postintervention data collection. The clinical trials commenced at week zero $(0)$ at the hospital. The participants who were eligible, and willing to participate in the study were reviewed by the research assistant 1 (RA 1) for pre-identified parameters of breast cancer at stages I and II as well as evaluate their physical activity profile after obtaining their biodata. Participants were required to complete the Physical Activity Readiness Questionnaire which have been used in previous studies $[46,47]$ to assess their suitability for an exercise programme. A "yes" answer to any of the questions requires that approval be obtained from the participant's physician in order to take part. They were educated on the purpose/procedure for the study. For participants in the intervention (pedometer) group, a physiotherapist (RA2) administered the six-week pedometer-based walking programme according to the Trans-Theoretical Model (TTM) framework to ensure quality [48]. A follow-up home programme guideline was provided. The physiotherapist also gave them a follow-up guide on how to continue the programme at home using the standardized guideline. The Physiotherapist was not blinded to participant allocation but the assessor was.

\section{Instruments}

\section{- Physical activity measurement:}

\section{Wearable technology - Fitbit Charge HR}

Participants in the Intervention (pedometer) and control groups were provided with pedometers which they wore on their wrist. The pedometer step counts/day was measured with the Fitbit Charge HR (Fitbit International Ireland) (Plate 1). The choice of this technology was made because the inter-device reliability of the Fitbit is good in measuring steps at all levels of aggregation (minutes, hours, days), but especially when steps were measured per day, and therefore individuals can reliably relate their daily physical activity scores with peers [61]. Furthermore, a systematic review on walking- and running-based pedometer trials indicated consistently high inter-device reliability for steps (Pearson and intraclass correlation coefficient - CC 0.76-1.00), distance (Interclass CC 0.90-0.99), and energy expenditure (Pearson and intraclass CC 0.71-0.97) with Fitbit [62]. To the researcher's knowledge, this model of pedometer has been utilized and validated in many previous intervention studies. In fact, good interdevice reliability was reported in seven studies using the Fitbit [62]. The Fitbit charge HR has several features beneficial to an intervention of this nature, including a cover to prevent accidental resetting, continuous heart rate measurement for 24 hours, tracks all physical activities in a day by providing number of steps, distance covered, floors climbed, calories burned, active minutes, time spent, hours slept, 
time awakened, sleep quality, display the statistics in wirelessly synchronized smartphones/computer monitors, and a 7-day memory which negates the need for participants to record their own step-counts.

\section{- 2. IPAQ:}

The International Physical Activity Questionnaire-short form (IPAQ-SF) is a subjective measure of physical activity was used to examine changes in modes of physical activity that would not be measured by the pedometer such as swimming or other forms of structured sport or exercise. Thus, daily physical activity was also measured using the IPAQ-SF [63]. The IPAQ-SF is a valid and reliable seven-item tool applied in measuring self-reported physical activity in the last seven days $[63,64]$.

It collects information about moderate and vigorous physical activity across four domains: work-related, transportation, housework/gardening, and leisure-time physical activity. The detail on specific physical activity domains, therefore, enabled the identification of specific domains of physical activity where changes may have occurred. Walking time is also included for the work, transport and leisure domains. Two additional questions measured time spent sitting which can be used as an indication of sedentary time. This questionnaire asks about three specific types of activity, namely walking, moderate-intensity activities, and vigorous-intensity activities [63]. The minutes spent every week on each type of activity were computed separately by multiplying the duration and frequency of activity [65]. A continuous activity score was calculated by multiplying the selected metabolic equivalent (MET) value and weekly minutes of activity, therefore expressing physical activity as MET-min per week [66]. The MET values and the levels of physical activity were calculated according to the guidelines for data processing and analysis of the IPAQ-SF [66] [67]. Criterion validity of IPAQ-SF had a median of about 0.30 , which was comparable to most other self-report validation studies [67]. Test-retest reliability data for the IPAQ-SF using Spearman correlation coefficients across 12 countries ranged from 0.96 (USA2) to 0.46 (SA Ru), but most were around 0.8 [63].

\section{- Body composition/Anthropometry measurement:}

Body anthropometry was measured while the participants were standing, wearing light clothing and no shoes.

i. Height The height was measured as the distance from the height scale platform to the vertex of the head using a stadiometer (Sartorius, AG Gottingen). The height was then, read-off, and recorded to the nearest 1.0 the centimetres [68].

ii. Body mass index: The Fat Loss Monitor with Scale (OMRON® HBF-400) was used to measure the body weight in $\mathrm{Kg}$. The body mass index was also calculated as the square of height $(\mathrm{m}) /$ weight (kg). [69].

iii. Waist-hip-ratio: The Waist and hip circumference were measured using a SECA 200 (SECA, Birmingham, UK) measuring tape. The waist-hip-ratio was calculated from these measurements. 
iv. Body fat mass; The percentage body fat mass was estimated using the Fat Loss Monitor with Scale (OMRON® HBF-400. Though the DEXA (Dual Energy X-Ray Absorptiometry) has been the established method for the accurate evaluation of body composition. However, OMRON has used research information from several hundred people from 10 to 80 years of age using the DEXA method to develop the method by which the Fat Loss MONITOR with Scale works. The body fat mass and body fat percentage are quantified by a method that includes five factors: electrical resistance, height, weight, age and gender. Body fat percentage was measured from $5-60 \%$ in $0.1 \%$ increments [69].

\section{- 3. Serum level of Superoxide dismutase:}

Laboratory study of all participants was done by a trained laboratory scientist. At baseline and end of week 6 , each participant's blood ( $5 \mathrm{mls}$ ) was drawn from the subcutaneous vein of the arm following standard blood sample collection procedures. Subsequently, the collected blood samples were stored in Serum Separator Tube (SST) without an anticoagulant, thereby allowing the clotting of the blood sample. This facilitated the separation of the serum from blood cells. The blood sample was kept at $40^{\circ} \mathrm{C}$ prior to centrifugation (3000 rpm, $10 \mathrm{~min}, 0-40 \mathrm{C}$ ). The serum level of Superoxide dismutase was measured by the procedure described by the manufacturer of the kit (BioAssay Systems, USA). A clear flat-bottom 96 well plate was used which contained serum samples as dilutes, 1:5 prior to assay. Thereafter, $20 \mu \mathrm{L}$ superoxide dismutae standards and samples were put into separate wells of the 96-well plate, with a clear flatbottom, into which $160 \mu \mathrm{L}$ working reagent $(160 \mu \mathrm{L}$ Assay Buffer, $5 \mu \mathrm{L}$ Xanthine and $5 \mu \mathrm{L}$ WST-1) was added and mixed thoroughly. Subsequently, $20 \mu \mathrm{L}$ of diluted XO enzyme (1:20 in diluent) was rapidly put into the plate using a multichannel pipette, and mixed. The optical density (OD) of the mixture was read instantly at $440 \mathrm{~nm}$ (OD0). The clear flat-bottom 96-well plate was at that point incubated at 60 minutes in the dark, at room temperature. The mixture was again read after incubation at OD 440nm (OD60). For each standard and sample, we calculated $\triangle$ OD60 =OD60 - ODO. A standard curve was plotted and utilised to estimate the sample concentration. Superoxide dismutase was measured in $(\mathrm{U} / \mathrm{ml})$.

\section{Covariates}

Covariates assessed and documented at baseline included: marital status, (single, married, widowed); occupation (Housewife, Farmer, Civil servant, Trader, Student). Breast cancer stage at diagnosis, family history of cancer, and treatment received - surgery (had surgery - Lumpectomy and Partial mastectomy; or no surgery; chemotherapy; and radiotherapy) were reported at initial review to assess eligibility prior to study enrolment.

\section{Follow-up:}

As the primary outcome measure of this study is physical activity level, strict follow-up on physical activity behavioural pattern was indicated. After completion of the six-week pedometer-based walking programme, physiotherapists set the optimal exercise level that should be adhered to during the follow-up periods. Over the follow-up period, participants were evaluated by their physiotherapist and medical oncologist at six weeks, twelve weeks and six months. At each assessment session, participants' history 
was obtained and likewise their physical activity level, antioxidant (superoxide dismutase) level and body composition. Any treatment administered to any breast cancer survivor for second, third or subsequent recurrence of breast cancer was appropriately documented. All the collected data were recorded on a follow-up Case Record Form (CRF).

\section{Data Analysis}

Data were analyzed using XLSTAT statistical and data analysis solution software (Boston, USA). Descriptive statistics were used to present the data in tables as frequency and percentages. Data collected were tested for normality using the Kurtosis (Fisher) Normality test. Subsequently, baseline differences in steps/day, physical activity level (MET-min/week), body composition (waist-to-hip ratio, percentage body fat mass and body mass index) and serum level of Superoxide dismutase between the intervention and control groups were tested for statistical significance using independent t-test. Statistical significance is defined as $p<0.05$ for all tests with data presented as mean and standard deviation.

\section{Ethical consideration:}

All methods were performed in accordance with the relevant guidelines and regulations of the University of Nigeria Health Research Ethics Committee, and in accordance with the Declaration of Helsinki.

\section{Results}

\section{Participants}

Overall, 128 Breast cancer survivors indicated interest to participate in the study over a 6-week public campaign. After screening them (Figure 1), 30 participants were excluded for reasons that include not meeting the inclusion criteria $(n=16)$, declining to participate $(n=9)$, and failing the physical activity readiness questionnaire $(n=5)$. Subsequently, the remaining 98 participants who were eligible, and willing to participate in the study were reviewed and recruited into the study on 27 July, 2016, by the research assistant 1 (RA 1) for pre-identified parameters of breast cancer at stages I and II as well as evaluate their physical activity profile after obtaining their biodata (Table 2). Over the follow-up period, the participants were evaluated by the physiotherapist (assessor) and medical oncologist at six weeks (of which the first follow-up visit was on 19 October, 2016), twelve weeks (second follow-up visit was on 11 January, 2017) and six months (third follow-up visit was on 5 April, 2017). Out of 98 participants that started the study, 10 (4 participants from the intervention arm and 6 participants from the control arm) were lost to followup due to health issues arising from surgery and side effects of chemotherapy. Thus, the attrition rate was $10.20 \%$. Five women from the intervention arm had missing data at weeks 3,5 and 6 , while three women from the control group had missing data at weeks 4-6 because they lacked electricity to charge their battery. Weekly downloaded data from the Fitbit server showed that 29 participants in the intervention group adhered to the walking plan in Table 1 and met the set biweekly target of 1500 step counts/day, and also attended all the consultation sessions. The adherence rate was thus determined as 
72.5\%. The participants flow through the study is presented in the Consolidated Standards of Reporting Trials (CONSORT) diagram (Figure 1).

Table 2

\begin{tabular}{|c|c|}
\hline Variables & Frequency \\
\hline Diagnosis duration (weeks) $(X \pm)$ & $6.8 \pm 31.5$ \\
\hline \multicolumn{2}{|l|}{ Breast cancer stage $(\mathrm{N}=80)$} \\
\hline - Stage I & $60(75)$ \\
\hline - Stage II & $20(25)$ \\
\hline Indicated for Surgery & $80(100)$ \\
\hline Had surgery & $71(88.75)$ \\
\hline - Lumpectomy & $11(13.75)$ \\
\hline - Partial mastectomy & $69(86.25)$ \\
\hline \multicolumn{2}{|l|}{ On Chemotherapy $(\mathrm{n}=80)$} \\
\hline - Yes & $80(100)$ \\
\hline \multicolumn{2}{|l|}{ On Radiotherapy } \\
\hline - Yes & $38(23.75)$ \\
\hline \multicolumn{2}{|l|}{ Menopausal status } \\
\hline - Postmenopausal & $51(63.75)$ \\
\hline \multicolumn{2}{|l|}{ Used oral contraceptives } \\
\hline - Yes & $20(25)$ \\
\hline \multicolumn{2}{|l|}{ Had a family history of cancer } \\
\hline - Yes & $24(30)$ \\
\hline
\end{tabular}

The majority of the breast cancer survivors were postmenopausal women, had mastectomy, chemotherapy and had never used oral contraceptives (Table 2). However, less than half of them have a family history of cancers and received radiotherapy. The overall mean values of the variables of interest among the 80 participants (Table 3 ) are as follows: - physical activity level - 983.92 711.01 (MET$\mathrm{min} /$ week); step count - $11,113.5 \pm 2438.21$ step count/day; waist-to-hip ratio - $0.86 \pm 0.18$; percentage body fat mass $-29.12 \pm 4.71 \%$; body mass index $-26.79 \pm 4.46 \mathrm{Kg} / \mathrm{m}^{2}$; serum level of Superoxide 
dismutase $-17.23 \pm 1.54 \mathrm{U} / \mathrm{mgHb}$; and age $-47.5 \pm 6.97 y$ ears. Their mean diagnosis duration was $6.8 \pm 31.5$ weeks.

Table 3

Socio-demographics, Physical activity level, body composition and serum concentration of superoxide dismutase of participants (intervention and control groups) $(\mathrm{N}=80)$

\begin{tabular}{|c|c|c|c|c|}
\hline Group & intervention Group & Control Group (N/\%) & Mean diff & p-value \\
\hline \multicolumn{5}{|l|}{ Marital status (N/\%) } \\
\hline - Single & $0(0)$ & $0(0)$ & & \\
\hline - Married & $49(61.25)$ & $50(62.50)$ & & \\
\hline - Widowed & $20(25.00)$ & $7(8.75)$ & & \\
\hline - Divorced & $0(0.00)$ & $8(10.00)$ & & \\
\hline Total & $80(100 \%)$ & $80(100 \%)$ & & \\
\hline \multicolumn{5}{|l|}{ Occupation (N/\%) } \\
\hline - House wife & $16(20.00)$ & $18(22.50)$ & & \\
\hline - Farmer & $0(0.00)$ & $12(15.00)$ & & \\
\hline - Civil servant & $32(40.00)$ & $28(47.50)$ & & \\
\hline - Trader & $23(35.00)$ & $12(15.00)$ & & \\
\hline - Student & $4(05.00)$ & $0(0.00)$ & & \\
\hline Total & $80(100)$ & $80(100)$ & & \\
\hline $\operatorname{BMI}\left(\mathrm{Kg} / \mathrm{m}^{2}\right)$ & $27.00(1.67)$ & $26.58(4.01)$ & 0.42 & 0.39 \\
\hline WHR & $0.83(0.05)$ & $0.89(0.24)$ & 0.05 & 0.18 \\
\hline$\%$ BFM & $28.77(2.71)$ & $29.47(4.85)$ & 0.7 & 0.32 \\
\hline PAL(MET-min/week) & $1007.80(518.03)$ & $960.05(841.63)$ & 47.75 & 0.42 \\
\hline Step count/day & $11468 \pm 2745.82$ & $10759 \pm 2097.27$ & 709 & 0.18 \\
\hline $\mathrm{SOD}(\mathrm{U} / \mathrm{mgHb})$ & $19.74(0.82)$ & $17.34(1.60)$ & 0.0235 & 0.41 \\
\hline
\end{tabular}

The intervention and control groups (Table 3 ) showed some similarities in their demographic characteristics. For instance, in each of the two groups, the majority of the participants were civil servants and married. However, the participant's age range and mean age showed that the those in the intervention 
group were relatively older (age range $=35-55$ years, $48.5 \pm 6.9 .05 y e a r s$ ) than the control group (age range $=41-65$ years, $50.5 \pm 6.62$ years $)$. but not significantly different $(p=0.22)$.

\section{Physical activity profile before and after pedometer intervention}

At baseline, the physical activity level/profile (MET-min/week) of the pedometer and control groups was similar as $20 \%$ (high), $40 \%$ (moderate) and $40 \%$ (low). (Table 3 ). There was no significant baseline difference $(p>0.05)$ in the physical activity profile/level (MET-min/week) and likewise the pedometer step count/day between the groups (Table 3), respectively. A post intervention comparison of the groups showed a significant increase in the physical activity profile/levels (MET-min/week) $(p=0.006, d=0.85$, change factor $=764.92 \%)$, and pedometer step count/day $(p=0.0002, d=0.80$, change factor $=295.8 \%)$ of the intervention group compared to control group (Table 4). The effect size was also large effect size. However, the change in the pedometer Step count/day was superior to physical activity levels (MET$\mathrm{min}$ /week). Furthermore, it was observed that the pre-intervention and post-intervention differences in the physical activity level (MET-min/week) between the groups was 47.75 MET-min/week and 413.4 MET-

$\mathrm{min} /$ week, respectively. Similarly, the differences in the steps/day between the groups was 709 steps/day and 2806.5 steps/day before and after the intervention, respectively. Therefore, the ratio of the differences in physical activity level (MET-min/week) and step count between the intervention group and control is determined as $47.75 \mathrm{MET}$-min/week divided by 709 steps/day (i.e., 1:15) at baseline; and 413.4 divided by 2806.5 (i.e., 1:7) at post-intervention, respectively. Based on the change factor (i.e., percentage change from the baseline value), the intervention group recorded $6.5 \%$ in physical activity level (MET$\mathrm{min} /$ week) and $4.6 \%$ increase in their step count/day compared to the control. 
Changes in physical activity, body composition and superoxide dismutase in pedometer group $(\mathrm{N}=40)$ and control $(\mathrm{N}=40)$ after 6 -week trials

\begin{tabular}{|c|c|c|c|c|c|c|}
\hline Variables & Intervention & Control & $\begin{array}{l}\text { Mean } \\
\text { diff }\end{array}$ & $\begin{array}{l}\text { Change } \\
\text { factor (\%) }\end{array}$ & $\begin{array}{l}\text { Effect } \\
\text { size }\end{array}$ & p-value \\
\hline \multicolumn{7}{|l|}{$\begin{array}{l}\text { PAL(MET- } \\
\text { min/week) }\end{array}$} \\
\hline & $1167.25 \pm 601.26$ & $753.8 \pm 369.24$ & 413.45 & $865.9 \%$ & 0.85 & $0.006 * \star$ \\
\hline \multicolumn{7}{|l|}{$\begin{array}{l}\text { Step } \\
\text { count/day }\end{array}$} \\
\hline & $12837 \pm 2572.90$ & $10030.5 \pm 1880.74$ & 2806.5 & $395.8 \%$ & 0.80 & $0.0002^{\star * *}$ \\
\hline \multicolumn{7}{|l|}{ WHR } \\
\hline & $0.79 \pm 0.08$ & $1.03 \pm 0.34$ & 0.24 & $450.5 \%$ & 1.13 & $0.002^{\star \star}$ \\
\hline \multicolumn{7}{|l|}{$\% B F M$} \\
\hline & $26.79 \pm 4.82$ & $30.32 \pm 4.94$ & 3.53 & $504.3 \%$ & 0.72 & $0.01 *$ \\
\hline \multicolumn{7}{|c|}{ BMI $\left(\mathrm{Kg} / \mathrm{m}^{2}\right)$} \\
\hline & $25.13 \pm 3.53$ & $26.71 \pm 3.71$ & -1.575 & $375.0 \%$ & 0.43 & 0.09 \\
\hline \multicolumn{7}{|l|}{$\begin{array}{l}\text { SOD } \\
\text { (U/mgHb) }\end{array}$} \\
\hline & $3.12 \pm 0.30$ & $2.62 \pm 0.38$ & 0.497 & $2114.9 \%$ & 1.01 & $<.0001^{\star * *}$ \\
\hline \multicolumn{7}{|c|}{ 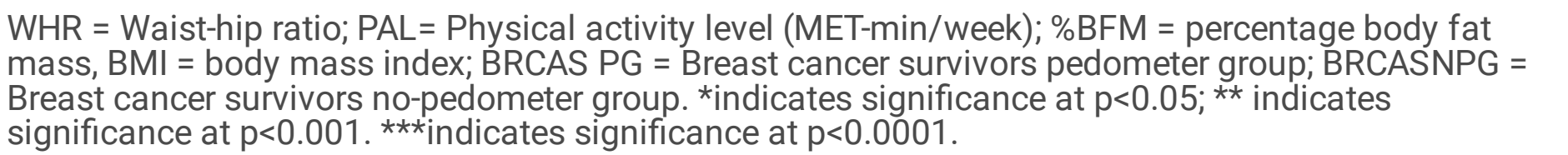 } \\
\hline
\end{tabular}

\section{Body compositions before and after pedometer intervention}

From all the indices of body composition at baseline, (except body mass index), half (50\%) of the participants (i.e., both pedometer and control groups) were either overweight or obese. However, based on the body mass index values, $52.5 \%(n=42)$ were either overweight or obese (Table 4). From the assessment of the body anthropometry using waist-hip-ratio, percentage body fat mass and body mass index as categorisation indices, $50 \%, 45 \%$ and $55 \%$ of those in the intervention group were overweight, respectively. In contrast, $30 \%, 30 \%$ and $35 \%$ of those in the control group were overweight (Table 5). Similarly, $15 \%, 10 \%$ and $0 \%$ of the participants in the intervention group were obese, while $5 \%, 15 \%$ and $5 \%$ of those in the control group were obese, respectively. There was no significant difference $(p>0.05)$ in the waist-to-hip ratio, percentage body fat mass and body mass index of the intervention group when 
compared to the control group at baseline (Table 4). However, a post-intervention assessment of the groups (Table 4) showed a significant decrease in the waist-to-hip ratio $(p=0.002, d=1.13$, change factor $=$ $350.5 \%)$, and percentage body fat mass $(p=0.01, d=0.72$, change factor $=410.0 \%)$ in the intervention group compared to control. The effect size was also large effect size, respectively, unlike body mass index $(\mathrm{p}=0.09, d=0.43$, change factor $=275.0 \%)$. 
Table 5

Descriptive analysis of changes in Physical activity level, and body composition among the BRCAS after 6-week trials

\begin{tabular}{|c|c|c|c|c|c|c|c|}
\hline \multirow{3}{*}{$\begin{array}{l}\text { Variables } \\
\text { Waist-hip } \\
\text { ratio }\end{array}$} & \multirow{3}{*}{$\begin{array}{l}\text { No of } \\
\text { categories }\end{array}$} & \multirow[t]{3}{*}{ Mode } & \multirow{3}{*}{$\begin{array}{l}\text { Mode } \\
\text { freq }\end{array}$} & \multicolumn{4}{|c|}{ Categories } \\
\hline & & & & \multicolumn{4}{|c|}{ Rel. frequency per category (\%) } \\
\hline & & & & Obese & Overweight & Normal & $\begin{array}{l}\text { Under- } \\
\text { weight }\end{array}$ \\
\hline $\begin{array}{l}\text { Intervention } \\
\text { group } \\
(\mathrm{N}=40)\end{array}$ & 3 & Overweight & 20 & $6(15)$ & $20(50)$ & $14(35)$ & 0 \\
\hline $\begin{array}{l}\text { Control } \\
\text { Group } \\
(\mathrm{N}=40)\end{array}$ & 3 & Normal & 26 & $2(5)$ & $12(30)$ & $26(65)$ & 0 \\
\hline $\begin{array}{l}\% \text { of body } \\
\text { fat mass }\end{array}$ & & & & Obesity & Overweight & Average & Fitness \\
\hline $\begin{array}{l}\text { Intervention } \\
\text { group } \\
(\mathrm{N}=40)\end{array}$ & 3 & Overweight & 18 & $4(10)$ & $18(45)$ & $14(35)$ & $4(10)$ \\
\hline $\begin{array}{l}\text { Control } \\
\text { Group } \\
(\mathrm{N}=40)\end{array}$ & 4 & Average & 20 & $6(15)$ & $12(30)$ & $20(50)$ & $2(5)$ \\
\hline $\begin{array}{l}\text { Body mass } \\
\text { index } \\
\left(\mathrm{Kg} / \mathrm{m}^{2}\right)\end{array}$ & & & & Obese & Overweight & Normal & $\begin{array}{l}\text { Under- } \\
\text { weight }\end{array}$ \\
\hline $\begin{array}{l}\text { Intervention } \\
\text { group } \\
(\mathrm{N}=40)\end{array}$ & 3 & Overweight & 22 & $0(0)$ & $28(70)$ & $12(30)$ & $0(0)$ \\
\hline $\begin{array}{l}\text { Control } \\
\text { Group } \\
(\mathrm{N}=40)\end{array}$ & 3 & Normal & 24 & $2(5)$ & $14(35)$ & $24(60)$ & $0(0)$ \\
\hline $\begin{array}{l}\text { PAL(MET- } \\
\text { min/week) }\end{array}$ & & & & High & Moderate & low & 0 \\
\hline $\begin{array}{l}\text { Intervention } \\
\text { group } \\
(\mathrm{N}=40)\end{array}$ & 3 & Low/moderate & 16 & $8(20)$ & $16(40)$ & $16(40)$ & \\
\hline $\begin{array}{l}\text { Control } \\
\text { Group } \\
(\mathrm{N}=40)\end{array}$ & 3 & Low/Moderate & 16 & $8(20)$ & $16(40)$ & $16(40)$ & 0 \\
\hline $\begin{array}{l}\text { BRCAS PG = } \\
\text { pedometer g }\end{array}$ & st canc & ivors pedom & rou & AS & Breast c & survi & b- \\
\hline
\end{tabular}




\section{Superoxide dismutase before and after pedometer intervention}

There was no significant baseline difference in the serum level of Superoxide dismutase of the Breast cancer survivors in the intervention group compared to the control group (Table 3). However, the absolute baseline difference in the serum level of Superoxide dismutase for the breast cancer survivors was $0.0235(\mathrm{U} / \mathrm{mgHb})$ when the intervention and control groups were compared, and subsequently increased to $0.497(\mathrm{U} / \mathrm{mgHb})$ after the 6-week trials. This represents $610.0 \%$ increase in the serum level of Superoxide dismutase in the intervention group compared to the control after the trials. The difference in mean is significant (Table 4) with a large effect size $(p=0.0001, d=1.01$; $)$.

\section{Adverse events}

Eleven study participants ( 6 control and 5 experimental group) experienced some pain and discomfort when undergoing phlebotomy to collect blood samples for the investigations. Four ( 1 control and 3 experimental group) participants stated that they were embarrassed when completing the questionnaires especially when questions related to lifestyles, were asked. Another 7 ( 6 control and 1 experimental) participants reported a feeling of embarrassment during the assessment of body composition because they felt they gained weight. Fifteen ( 5 control and 10 experimental group) participants felt concern about their security while using the pedometer which they felt will relay their location to anyone who has access to the Fitbit sever centre apart from the investigators. One patient (experimental group) reported a fall on a wet ground and hit her arm on the railings of a walkway. She was rushed to the hospital and admitted for observation. There were no injuries recorded apart from minor bruises on the right arm. The participant's blood pressure was $120 / 82 \mathrm{mmHg}$, respiratory rate was 20 breaths $\mathrm{min}-1, \mathrm{SpO} 292 \%$ at 10 $L \cdot \min -102$, while the heart rate was 72 beats $\cdot \min -1$. The patient was discharged the next day without further complaints and completed the study. Two deaths were recorded ( 1 control and 1 experimental group) at the 12-week and 6-month follow-up time points, respectively. They both have a history of cardiovascular diseases and had fatal thrombotic events. Nine participants ( 3 control and 6 experimental) complained of musculoskeletal pain within the first week, which progressively reduced and resolved in the fourth week.

\section{Discussion}

The majority of the breast cancer survivors in this study were: married, postmenopausal, and mainly had sedentary jobs either as full-time housewives/civil servants/traders, all of which have been identified as key demographic/lifestyle characteristics of obese individuals and/or risk factors for breast cancer [7476]. Furthermore, the baseline body composition and physical activity lifestyle of the breast cancer survivors represent two major modifiable risk factors that may have implications for breast cancer prevention/recurrence and perhaps survival. Firstly, there is a prevalence of low physical activity profile in $40 \%(n=32)$ of the entire sample at baseline. The likely health implications may include weight gain, which may explain why the overall mean body mass index of all the participants was $26.79 \pm 4.50 \mathrm{Kg} / \mathrm{m}^{2}$ 
which indicates that they were overweight $\left(>25 \mathrm{Kg} / \mathrm{m}^{2}\right)$. Clinically, it should imply that on the average, adiposity was above the normal range in a reasonable number of the participants.

Similarly, the measured indices of body composition in this study revealed a clear and consistent pattern that raises a concern about the prevalence of high adiposity in breast cancer survivors. For instance, the mean waist-to-hip ratio of all the participants $(n=80)$ in both groups was $>0.85(0.86)$ which the WHO has determined as the borderline reference value for abdominal obesity in females [1]. This may have some health implications for breast cancer survivors since there is evidence of a strong association between the waist-to-hip ratio (waist-hip ratio) and the amount of weight gained over a woman's lifetime to the development of postmenopausal breast cancer [77]. However, the reasons for such weight gain may not be solely attributed to low physical activity or greater inactivity since weight gain is a common problem among postmenopausal women [69] and in individuals receiving adjuvant chemotherapy for breast cancer who have an average weight gain of $4.5 \mathrm{~kg}$ [31, 33]. In fact, most premenopausal women treated for breast cancer with cyclophosphamide, methotrexate, and fluorouracil gained an average of 2 to $6 \mathrm{~kg}$ $[31,33,78]$. The weight gain status is present despite majority $(60 \%)$ of the participants scoring high and moderate physical activity lifestyle, yet their mean body mass index and waist-hip-ratio indicate that they were overweight instead of normal/underweight. An explanation could be increase in fat mass, percentage body fat mass, and a decrease in lean muscle mass [25], which may alter upper and lower extremity muscle strength $[31,79]$ impair ambulation/walking and restrict physical activity behaviour. Consequently, strategies aimed at weight control in women treated for breast cancer should include other measures that may improve fat metabolism as well as recommendations for physical activity.

\section{Physical activity profile before and after pedometer intervention}

The significant increase in the step count/day of the intervention group compared to the control group demonstrates the efficacy of the pedometer-based physical activity promotion strategy in breast cancer survivors and may have clinical significance considering the large effect size. An analysis of the ratio of the differences in physical activity level (MET-min/week) and step count between the intervention group and control at baseline (1:15); and post-intervention (1:7), respectively, suggests that other (i.e., moderate + vigorous physical activity) components measured by IPAQ-SF apart from walking were improved among those that used the pedometer. The IPAQ measures physical activity tasks (walking + moderateintensity activity + vigorous-intensity activity) and the pedometer measures/quantifies the walking component of the physical activity tasks, the higher percentage change in physical activity level (MET$\mathrm{min} /$ week $=764.92 \%$ ) recorded among the breast cancer survivors, and which occurred when there was a lesser percentage change in walking (step count $=395.8 \%$ ) activity. The increased physical activity level suggest that an increase in either the moderate-intensity activity component or vigorous-intensity activity component or both components of the physical activity tasks. It is possible that the pedometer-based walking programme improved participants' walking behaviour/physical activity. This may have helped them regain greater levels of physical fitness, endurance and capacity to engage in either the moderate or 
vigorous physical activity, which ordinarily, they seemed incapable of considering the baseline data. An explanation could be that individuals are more likely to attain public health guidelines by walking at least 8000 steps/day [80]. There is evidence that when steps are above 8000 steps/day, it triggers positive effects on conventional metabolic parameters, such as blood pressure, and waist circumference [81, 82].

\section{Body compositions before and after pedometer intervention}

Interpreting the change factor (i.e., percentage change from the baseline value), it was evident that the breast cancer survivors recorded decreases in percentage body fat mass $(12.18 \%)$, waist-to-hip ratio (6.88\%), and body mass index (6.93\%), respectively. The reduction in body mass index was not significant. One reason for this observation might be related to the fact that physical activity tends to increase lean muscle mass which increases the value of body mass index but reduces adiposity [83]. Therefore, the insignificant decrease in body mass index may not suggest an insignificant decrease in adiposity. Therefore, the body mass index may not be a good measure of adiposity, and its interpretation should be done with reference to other indices such as measures of central and general adiposity which was done in this study. Though the reduction in the body mass index was not significant, yet it is an important trend, which was observed in this study given that the body mass index of the intervention group decreased from about $27 \mathrm{Kg} / \mathrm{m}^{2}$ to about $25 \mathrm{Kg} / \mathrm{m}^{2}$. Previous prospective study of women with operable breast cancer concluded that women with a body mass index of 20 to $25 \mathrm{~kg} / \mathrm{m}^{2}$, had a low risk of recurrent breast cancer [84]. However, the risk of recurrence rose steadily when body mass index levels increased beyond $25 \mathrm{~kg} / \mathrm{m}^{2}$. Therefore, the reduction of body mass index of the breast cancer survivors to $25 \mathrm{Kg} / \mathrm{m}^{2}$ in this study should likewise hold a promise of reducing the risk of recurrent breast cancer, which is of clinical importance. Similarly, by attaining a body mass index of $25 \mathrm{Kg} / \mathrm{m}^{2}$, it is demonstrated that there was a re-compartmentalization of the body composition through weight reduction in the intervention group. Already, recent epidemiologic data have established a link between excess body weight and breast cancer development and subsequent recurrence $[77,85,86]$. Similar trends were observed with other indices of body composition (waist-hip-ratio and percentage body fat mass), which may have some clinical implications. For instance, the waist-to-hip ratio is a measure of abdominal/visceral/central obesity [25], and has been identified as the greatest risk of breast cancer [87]. Consequently, the significant reduction of the waist-to-hip ratio in the intervention group than the control reflects a reduction in adiposity, which may reduce cancer recurrence.

The relationship established between pedometer-based walking and body composition in this study supports the findings of previous studies that increasing the level of physical activity influences body composition by minimizing adiposity while increasing the free fat mass [88-91]. The reduction of adiposity may ameliorate the inflammatory micro-environment and enhance the antioxidant activity in breast cancer survivors, which otherwise would have mediated breast cancer recurrence in survivors. This view is supported by evidence that central adiposity may induce breast cancer by suppressing the antioxidant activity, which may encourage the accumulation of reactive oxygen species [25] likely to encourage oxidative stress as the critical step for cancer induction. Usually, this is an outcome of an inactive lifestyle, which promotes the accumulation of visceral fat, with subsequent adipose tissue 
infiltration by pro-inflammatory immune cells, increased release of adipokines and the development of a low-grade systemic inflammatory state $[92,93]$. For this reason, also, excessive body weight, particularly obesity (which was observed to increase among control group (since percentage body fat increased from $29.47 \pm 4.85 \%$ to $30.32 \pm 4.94$, and waist-hip-ratio increased from $0.89 \pm 0.24$ to $1.03 \pm 0.34$ ), has been defined as an inflammatory state $[89,92]$. The low-grade systemic inflammation associated with obesity has also been associated with the development of insulin resistance, atherosclerosis, neurodegeneration and tumour growth [94-96].

An exploration of the baseline and post-intervention data revealed changes in the indices of body composition (percentage body fat mass, body mass index and waist-to-hip ratio), physical activity level (Met-min/week and the number of step count/day), and serum level of superoxide dismutase in the breast cancer survivors. Based on the between group analysis, the change factor, (i.e., percentage change from the baseline value) revealed that the intervention group recorded a $610 \%$ increase in the serum level of Superoxide dismutase (antioxidant) compared to control. Invariably, the pedometer-based walking programme significantly boosted the serum level of Superoxide dismutase $(p=<.0001)$ compared to control. Therefore, there may be a relationship between physical activity and serum superoxide dismutase in breast cancer survivors. There is evidence in studies of murine models that physical exercises, especially aerobic exercises, (reduced leptin, and interleukin-6, improved insulin resistance, and increased adiponectin) reversed weight gain, and obesity, attenuated tumour burden and cachexia, decreased growth rate and final weight of the tumour, improved body composition, and physical fitness [97-101]. Therefore, physical activity intervention may have a defined molecular basis for its positive effects on superoxide dismutase considering the highlighted ameliorative effects on the pro-inflammatory cytokines, leptin, and insulin action.

\section{Conclusion}

\section{Implications for practice}

In breast cancer survivors who have undergone chemotherapy, there is a possibility of significant distortion/decline in the measures of body composition, physical activity lifestyle and antioxidant level/activity. The research hypothesis was also tested and suggest that 6-week pedometer-based physical activity lifestyle significantly boosted antioxidant activity, improved body composition (except the body mass index) and physical activity level in breast cancer survivors.

It was shown that a 6-week pedometer use, increased the step count/day by 2806.5 . Similarly, 2806.5 step counts/day resulted in: 0.2365 decrease in waist-hip-ratio, $3.53 \%$ decrease in percentage body fat mass, $1.575 \mathrm{Kg} / \mathrm{m}^{2}$ decrease in body mass index, and $0.497 \mathrm{U} / \mathrm{mgHb}$ increase in superoxide dismutase. Therefore, over the same period, an increase of 1000 step count/day should result to 0.08 decrease in waist-hip-ratio, $1.25 \%$ decrease in percentage body fat mass, $0.56 \mathrm{Kg} / \mathrm{m}^{2}$ decrease in body mass index, and $0.18 \mathrm{U} / \mathrm{mgHb}$ increase in superoxide dismutase. So, given the baseline step counts/day of $11468 \pm 2745.82$ and $10759 \pm 2097.27$ for the pedometer and control groups, with percentage body fat 
mass of $28.77 \pm 2.71$ and $29.47 \pm 4.85$, respectively, it is estimated that additional 3,000 step counts/day and 4000 step counts/day are respectively required to reduce their percentage body fat mass to a normal level ( $\leq$ over a 6-week period). Hence, with the pedometer, it is possible to determine or estimate the step counts/day that may be required to induce a molecular change in the antioxidant activity. Using the pedometer therefore allows the monitoring of step counts/day as a correlate of important underlying clinical events related to body fat distribution, fat metabolism, body composition and antioxidant level required for prevention of breast cancer recurrence which may have relevance in public health promotion.

Overall, there is an indication that pedometer-based walking programme could be an effective behavioural intervention that should be explored at the population level to promote participation in physical activity among breast cancer survivors. The results of this study agree with the findings of another recent clinical trial - the ACTIVATE Trial [102]. - which demonstrated that the use of wearable technology, such as the pedometer, offers an affordable opportunity that can be scaled-up to enable more active lifestyles for Breast cancer survivors. However, it is not clear to what extent this approach will translate to a sustainable lifestyle change since this study lasted only 6 weeks and should be the focus of future study.

\section{Implications for research}

Further studies should be conducted to determine the protective effects of other forms of exercises against breast cancer induction/recurrence, especially abdominal exercises, since it may ameliorate central adiposity, and thus may have implications for antioxidant activity and inflammation. This study did not include inflammatory biomarker, therefore, did not provide objective data on the likely antiinflammatory effects of pedometer-based physical activity promotion in this population, and is recommended for future studies. This study demonstrates that an unsupervised pedometer-based walking programme with right counselling and set goals can translate into tangible health benefits among breast cancer survivors. It also strengthens the evidence for the therapeutic utility of pedometers in promoting behavioural intervention strategies for improved health outcomes in this population. Therefore, the pedometer-based walking approach could be a novel strategy suitable for public health promotion in the post-corona virus disease (COVID-19) era where supervised gym classes are less advisable to contain infection. Thus, it is recommended that the effectiveness of pedometer-based walking programmes should be further investigated in population-based studies for breast cancer prevention.

\section{Strength and limitations of the study}

The attrition rate was less than $15 \%$ and minimizes the risk of follow-up bias. However, therapist and participant blinding were not possible due to the nature of the design, but will likely have no effect on the outcome measures which addresses the fear of performance bias. The intention to treat analysis was done and agrees with the general trends of the results. All these factors should minimize the risk of bias that may affect our confidence in the estimate of the effect of the intervention on the variables of interest compared to the control. Another strength of this study is that it relied on several measures of body 
composition with complementary strengths that provide insight beyond their areas of weaknesses. For instance, the percentage body fat mass is a measure of fitness level, since it is the only body measurement which directly calculates relative body composition without regard to height or weight. The widely used body mass index provides a measure that allows the comparison of the adiposity of individuals of different heights and weights. However, body mass index has high specificity and low sensitivity [83].

The major weakness of this study is that the inflammatory biomarkers were not studied to provide a fuller picture of how the antioxidant and anti-inflammatory effects of physical activity relate to the physical activity level and body fat distribution in Breast cancer survivors. This is important because breast cancer is a disease characterised by both oxidative reactions and inflammation [103], and both factors should be examined together in future studies.

\section{Adverse events}

The participants who experienced pain and mild discomfort during the phlebectomy did not show any serious adverse reactions or side-effects after the procedure. However, they were counselled and reassured by the Physician/physiotherapists of their safety after their vital signs and screening showed no other symptoms of serious adverse events. Four ( 1 control and 3 experimental group) participants who mentioned that they were embarrassed when completing the questionnaires especially when questions related to lifestyles and body composition assessment, were counselled on ways of developing positive self-image and esteem. The participants who were concerned about the privacy of the data collected with the pedometer were assured of the confidentiality of the information they provided to the investigators as well as information transmitted by the Fitbit pedometer to the server. They were also given demonstrations on how the Fitbit pedometer works to give them confidence that it does not collect information on participant' location. Moreover, the participants were also assured that the Fitbit server is secured and non-authorised individuals do not have access to information relayed to the server by the pedometer. The musculoskeletal events were threefold more in the intervention group than control. Though these musculoskeletal events were not serious, but seem to indicate that participants may benefit from physical activity counselling. This should include guidance/information on how to avoid injuries, and scale up walking programmes in a gradual manner. The deaths were unrelated to the walking programme but were mainly attributed to the complications arising from the cardiovascular events. Overall, there were no serious adverse events reported in this study. The experimental (Pedometerwalking) group showed a high adherence level with a low risk of serious adverse effects, which seem to imply that pedometer-based walking intervention was found safe and generally acceptable to the participants.

Clinical trials registry and Trials Registration number. Pan African Clinical Trials Registry - Trial ID: PACTR202001542824141.

\section{List Of Abbreviations}




\begin{tabular}{|ll|}
\hline Term & Definition \\
\hline VO2 max. & Maximal oxygen consumption \\
\hline SEER & Surveillance, Epidemiology, and End Results (SEER) \\
\hline MVPA & Moderate to vigorous physical activity \\
\hline IPAQ & The International Physical Activity Questionnaires \\
\hline IPAQ-SF & The International Physical Activity Questionnaire \\
\hline MET-min/week & metabolic equivalent minutes/week \\
\hline TTM & Trans-Theoretical Model \\
\hline CRF & Case record form \\
\hline DEXA & Dual Energy X-Ray Absorptiometry \\
\hline SST & Serum Separator Tube \\
\hline OD & optical density \\
\hline OD0 & optical density at zero minutes \\
\hline OD60 & optical density at sixty minutes \\
\hline RA & Research assistant \\
\hline CONSORT & Consolidated Standards of Reporting Trials \\
\hline CC & correlation coefficient \\
\hline COVID-19 & Corona virus disease \\
\hline IPAQ & International Physical Activity Questionnaire \\
\hline WHO & World Health Organisation \\
\hline
\end{tabular}

\section{Declarations}

\section{Ethics approval and consent to participate}

This University of Nigeria Health Research Ethics Committee approved this study NHREC/05/01/2008BParticipants' confidentiality was maintained by using code numbers instead of names and ensuring that records were destroyed at the end of the study. Participants gave their written informed consent, prior to participation and after the purpose of the study was explained to them. They were informed of their right to withdraw from the study at any time of their choice and these rights were strictly respected in accordance with the Helsinki declarations. 
Not applicable

\section{Availability of data and materials}

The datasets generated and/or analysed during the current study are not publicly available due their containing information that could compromise the privacy of research participants but are available from the corresponding author on reasonable request. The full Trials protocol can also be obtained at https://pactr.samrc.ac.za/Search.aspx. or from the corresponding author - Sam Chidi Ibeneme via email: sam.ibeneme@unn.edu.ng.

\section{Competing interests}

The Authors declare that there is no conflict of interest

\section{Funding}

This research received no specific grant from any funding agency in the public, commercial, or not-forprofit sectors

\section{Authors' Contributions}

SCI, ADE and GF conceived of the study, participated in the design of the study, field work, coordination, performed the statistical analysis and drafted the manuscript. EE, $A L, O O, A N$, and $I O$ participated in the fieldwork, design and coordination. HM and ATA helped to perform the statistical analysis and helped to draft the manuscript. All authors read and approved the final manuscript.

\section{Acknowledgments}

We wish to acknowledge that this work was presented at the World Physiotherapy Congress 2021. We wish to acknowledge the support of Hochschule Hannover University in providing the pedometers, and assistance of the Spectrum molecular laboratory, Enugu, Nigeria, in conducting the molecular laboratory studies.

\section{References}

1. World Health Organisation. Waist-Hip Ratio Report of a WHO Expert Consultation. Geneva: World Health Organisation; 2008. [Accessed.

2. Weisell RC. Body mass index as an indicator of obesity. Asia Pacific Journal of Clinical Nutrition. 2002;11(8):S681-S4. https://doi.org/10.1046/j.1440-6047.11.s8.5.x

3. World Health Organization. Programming of chronic disease by impaired fetal nutrition: evidence and implications for policy and intervention strategies. World Health Organization; 2001. [Accessed.

4. Lohman TG, Going SB. Body composition assessment for development of an international growth standard for preadolescent and adolescent children. Food and nutrition bulletin. 
2006;27(4_suppl5):S314-S25

5. Duman CH, Schlesinger L, Russell DS, Duman RS. Voluntary exercise produces antidepressant and anxiolytic behavioral effects in mice. Brain research. 2008;1199:148-58

6. Lubkowska A, Dudzińska W, Bryczkowska I, Dołęgowska B. Body composition, lipid profile, adipokine concentration, and antioxidant capacity changes during interventions to treat overweight with exercise programme and whole-body cryostimulation. Oxidative Medicine and Cellular Longevity. $2015 ; 2015$

7. Maleki BH, Tartibian B, Mooren FC, FitzGerald LZ, Krüger K, Chehrazi M, Malandish A. Low-tomoderate intensity aerobic exercise training modulates irritable bowel syndrome through antioxidative and inflammatory mechanisms in women: Results of a randomized controlled trial. Cytokine. 2018;102:18-25

8. Balekouzou A, Yin P, Afewerky HK, Bekolo C, Pamatika CM, Nambei SW, Djeintote M, Doui Doumgba A, Mossoro-Kpinde CD, Shu C. Behavioral risk factors of breast cancer in Bangui of Central African Republic: A retrospective case-control study. PLoS One. 2017;12(2):e0171154

9. Guthold R, Louazani SA, Riley LM, Cowan MJ, Bovet P, Damasceno A, Sambo BH, Tesfaye F, Armstrong TP. Physical activity in 22 African countries: results from the World Health Organization STEPwise approach to chronic disease risk factor surveillance. American journal of preventive medicine. 2011;41(1):52-60

10. Hou N, Ndom P, Jombwe J, Ogundiran T, Ademola A, Morhason-Bello I, Ojengbede O, Gakwaya A, Huo D. An epidemiologic investigation of physical activity and breast cancer risk in Africa. Cancer Epidemiology and Prevention Biomarkers. 2014;23(12):2748-56

11. Sobngwi E, Mbanya JCN, Unwin NC, Aspray TJ, Alberti K. Development and validation of a questionnaire for the assessment of physical activity in epidemiological studies in Sub-Saharan Africa. International Journal of Epidemiology. 2001;30(6):1361-8

12. Siegel R, Naishadham D, Jemal A. Cancer statistics, 2012. CA: a cancer journal for clinicians. 2012;62(1):10-29

13. Azubuike S, Hayes L, McNally R. Non-vigorous physical activity and the risk of breast cancer among Nigerian women. Annals of Oncology. 2019;30:iii61

14. Bigman G, Adebamowo SN, Jedy-Agba EE, Achusi IB, Adebamowo CA. Association of leisure time physical activity with breast cancer risk and breast cancer sub-types in Nigerian women. American Society of Clinical Oncology; 2019.

15. Odutola MK, Olukomogbon T, Igbinoba F, Otu TI, Ezeome E, Hassan R, Jedy-Agba E, Adebamowo SN. Cancers attributable to overweight and obesity from 2012 to 2014 in Nigeria: a population-based cancer registry study. Frontiers in oncology. 2019;9:460

16. Arowolo O, Olasehinde O, Oyekunle A, Adisa A, Alatise O, Adesunkanmi A. The influence of life behaviour, diet, physical activities on the incidence of breast cancer among women attending Obafemi Awolowo University Teaching Hospital Complex, Ile-Ife. Journal of Cancer Research and Experimental Oncology. 2018;10(1):1-9 
17. Azubuike S, Hayes L, McNally R. Occupational physical activity and the risk of breast cancer among Nigerian women. European Journal of Public Health. 2018;28(suppl_4):cky213. 447

18. Azubuike S, McNally R, Hayes L. Household, Moderate Physical Activity and the Risk of Breast Cancer Among Nigerian Women: An Epidemiological Study. American Society of Clinical Oncology; 2018.

19. Adegoke B, Oyeyemi A. Physical inactivity in Nigerian young adults: prevalence and sociodemographic correlates. Journal of physical activity and Health. 2011;8(8):1135-42

20. Sylla B, Wild C. Cancer burden in Africa in 2030: Invest today and save tomorrow. Journal Africain du Cancer/African Journal of Cancer. 2012;4(1):1-2

21. Sylla BS, Wild CP. A million Africans a year dying from cancer by 2030: what can cancer research and control offer to the continent? International journal of cancer. 2012;130(2):245-50

22. International Agency for Research on Cancer. Nigeria (Source: Globocan 2020): World Health Organisation; 2020 [Available from: https://gco.iarc.fr/today/data/factsheets/populations/566nigeria-fact-sheets.pdf.

23. Hanson $S$, Jones $A$. Is there evidence that walking groups have health benefits? A systematic review and meta-analysis. British journal of sports medicine. 2015;49(11):710-5

24. Hegberg NJ, Tone EB. Physical activity and stress resilience: Considering those at-risk for developing mental health problems. Mental Health and Physical Activity. 2015;8:1-7

25. Visovsky C. Muscle strength, body composition, and physical activity in women receiving chemotherapy for breast cancer. Integrative cancer therapies. 2006;5(3):183-91

26. US Department of Health and Human Services. Physical activity guidelines for Americans. Hyattsville: US Department of Health and Human Services; 2008.

[Accessed].https://health.gov/sites/default/files/2019-

09/Physical_Activity_Guidelines_2nd_edition.pdf.

27. Boyle T, Vallance JK, Ransom EK, Lynch BM. How sedentary and physically active are breast cancer survivors, and which population subgroups have higher or lower levels of these behaviors? Supportive Care in Cancer. 2016;24(5):2181-90

28. Phillips SM, Dodd KW, Steeves J, McClain J, Alfano CM, McAuley E. Physical activity and sedentary behavior in breast cancer survivors: new insight into activity patterns and potential intervention targets. Gynecologic oncology. 2015;138(2):398-404

29. Faghri P, Stratton K, Momeni K. Sedentary lifestyle, obesity, and aging: implication for prevention. J Nutr Disorders and Therapy. 2015;5(1)

30. Jebb SA, Moore MS. Contribution of a sedentary lifestyle and inactivity to the etiology of overweight and obesity: current evidence and research issues. Medicine and science in sports and exercise. 1999;31(11 Suppl):S534-41

31. Demark-Wahnefried W, Winer EP, Rimer BK. Why women gain weight with adjuvant chemotherapy for breast cancer. Journal of Clinical Oncology. 1993;11(7):1418-29 
32. Eliassen AH, Colditz GA, Rosner B, Willett WC, Hankinson SE. Adult weight change and risk of postmenopausal breast cancer. Jama. 2006;296(2):193-201

33. Van den Berg M, Kok D, Visser M, De Vries J, de Kruif JTC, De Vries Y, Posthuma L, Sommeijer D, Timmer-Bonte A, Los $\mathrm{M}$. Changes in body composition during and after adjuvant or neo-adjuvant chemotherapy in women with breast cancer stage I- IIIB compared with changes over a similar timeframe in women without cancer. Supportive Care in Cancer. 2020;28(4):1685-93

34. Shapiro CL, Recht A. Side effects of adjuvant treatment of breast cancer. New England Journal of Medicine. 2001;344(26):1997-2008

35. Chhaya SJ, Quiros-Molina D, Tamashiro-Orrego AD, Houlé JD, Detloff MR. Exercise-induced changes to the macrophage response in the dorsal root ganglia prevent neuropathic pain after spinal cord injury. Journal of neurotrauma. 2019;36(6):877-90

36. Ferioli M, Zauli G, Maiorano P, Milani D, Mirandola P, Neri LM. Role of physical exercise in the regulation of epigenetic mechanisms in inflammation, cancer, neurodegenerative diseases, and aging process. Journal of cellular physiology. 2019;234(9):14852-64

37. Brown WM, Davison GW, McClean CM, Murphy MH. A systematic review of the acute effects of exercise on immune and inflammatory indices in untrained adults. Sports medicine-open. 2015;1(1):1-10

38. Tvede N, Kappel M, Halkjoer-Kristensen J, Galbo H, Pedersen B. The effect of light, moderate and severe bicycle exercise on lymphocyte subsets, natural and lymphokine activated killer cells, lymphocyte proliferative response and interleukin 2 production. International journal of sports medicine. 1993;14(05):275-82

39. Sparling PB, Howard BJ, Dunstan DW, Owen N. Recommendations for physical activity in older adults. Bmj. 2015;350

40. Smith L, Ekelund U, Hamer M. The potential yield of non-exercise physical activity energy expenditure in public health. Sports medicine. 2015;45(4):449-52

41. Hillsdon $M$, Thorogood M. A systematic review of physical activity promotion strategies. British journal of sports medicine. 1996;30(2):84-9

42. Rafferty AP, Reeves MJ, McGee HB, Pivarnik JM. Physical activity patterns among walkers and compliance with public health recommendations. Medicine and science in sports and exercise. 2002;34(8):1255-61

43. Harris T, Kerry SM, Limb ES, Furness C, Wahlich C, Victor CR, Iliffe S, Whincup PH, Ussher M, Ekelund U. Physical activity levels in adults and older adults 3-4 years after pedometer-based walking interventions: Long-term follow-up of participants from two randomised controlled trials in UK primary care. PLoS medicine. 2018;15(3):e1002526

44. Zhong B. How to calculate sample size in randomized controlled trial? Journal of thoracic disease. 2009;1(1):51

45. Demark-Wahnefried W, Clipp EC, Lipkus IM, Lobach D, Snyder DC, Sloane R, Peterson B, Macri JM, Rock CL, McBride CM. Main outcomes of the FRESH START trial: a sequentially tailored, diet and 
exercise mailed print intervention among breast and prostate cancer survivors. Journal of Clinical Oncology. 2007;25(19):2709-18

46. Thomas S, Reading J, Shephard RJ. Revision of the physical activity readiness questionnaire (PARQ). Canadian journal of sport sciences. 1992

47. Warburton DE, Jamnik VK, Bredin SS, Gledhill N. The physical activity readiness questionnaire for everyone (PAR-Q+) and electronic physical activity readiness medical examination (ePARmed-X+). The Health \& Fitness Journal of Canada. 2011;4(2):3-17

48. Aittasalo M, Miilunpalo S, Suni J. The effectiveness of physical activity counseling in a work-site setting: A randomized, controlled trial. Patient Education and Counseling. 2004;55(2):193-202

49. Marcus BH, Simkin LR. The transtheoretical model: applications to exercise behavior. Medicine \& Science in Sports \& Exercise. 1994

50. Hutchison AJ, Breckon JD, Johnston LH. Physical activity behavior change interventions based on the transtheoretical model: a systematic review. Health Education \& Behavior. 2009;36(5):829-45

51. Kirk A, Barnett J, Mutrie N. Physical activity consultation for people with Type 2 diabetes. Evidence and guidelines. Diabetic Medicine. 2007;24(8):809-16

52. Loughlan C, Mutrie N. Recruitment of sedentary NHS staff for a workplace exercise programme using an adapted "stages of change" exercise questionnaire. J Sports Sci. 1995;13:63-4

53. Lowther M, Mutrie N, Scott E. Promoting physical activity in a socially and economically deprived community: a 12 month randomized control trial of fitness assessment and exercise consultation. Journal of Sports Sciences. 2002;20(7):577-88

54. Mutrie N, Maclntyre P, Kirk A, Fisher M. Effects of a 12-month physical activity counselling intervention on glycaemic control and on the status of cardiovascular risk factors in people with Type 2 diabetes. Diabetologia. 2004;47(5):821-32

55. Rollnick S, Miller WR, Butler C. Motivational interviewing in health care: helping patients change behavior: Guilford Press; 2008.

56. Ogilvie D, Foster CE, Rothnie H, Cavill N, Hamilton V. Interventions to promote walking: systematic. BMJ. 2007;334(7605)

57. Tudor-Locke C, Sisson SB, Collova T, Lee SM, Swan PD. Pedometer-determined step count guidelines for classifying walking intensity in a young ostensibly healthy population. Canadian journal of applied physiology. 2005;30(6):666-76

58. World Health Organization. Global strategy on diet, physical activity and health. World Health Organization; 2004. [Accessed].https://apps.who.int/iris/handle/10665/43035.

59. Baker G, Mutrie N, Lowry R. Using pedometers as motivational tools: Are goals set in steps more effective than goals set in minutes for increasing walking? International Journal of Health Promotion and Education. 2008;46(1):21-6

60. Borg G. Borg's perceived exertion and pain scales: Human kinetics; 1998. 
61. Dontje ML, De Groot M, Lengton RR, Van Der Schans CP, Krijnen WP. Measuring steps with the Fitbit activity tracker: an inter-device reliability study. Journal of medical engineering \& technology. 2015;39(5):286-90

62. Evenson KR, Goto MM, Furberg RD. Systematic review of the validity and reliability of consumerwearable activity trackers. International Journal of Behavioral Nutrition and Physical Activity. 2015;12(1):1-22

63. Craig CL, Marshall AL, Sjöström M, Bauman AE, Booth ML, Ainsworth BE, Pratt M, Ekelund U, Yngve A, Sallis JF. International physical activity questionnaire: 12-country reliability and validity. Medicine \& science in sports \& exercise. 2003;35(8):1381-95

64. Lee PH, Macfarlane DJ, Lam TH, Stewart SM. Validity of the international physical activity questionnaire short form (IPAQ-SF): A systematic review. International Journal of Behavioral Nutrition and Physical Activity. 2011;8(1):1-11

65. IPAQ Research Committee. Guidelines for data processing and analysis of the International Physical Activity Questionnaire (IPAQ)-short and long forms 2005 [Available from: http://www. ipaq. ki. se/scoring. pdf.

66. IPAQ. International Physical Activity Questionnaire [Available from: https://sites.google.com/site/theipaq/scoring-protocol.

67. Hagströmer M, Oja P, Sjöström M. The International Physical Activity Questionnaire (IPAQ): a study of concurrent and construct validity. Public health nutrition. 2006;9(6):755-62

68. Fahey bTD, Insel PM, Roth WT, Insel CEA. Fit \& Well: Core Concepts and Labs in Physical Fitness and Wellness Loose Leaf. California: Mayfield Publishing Company; 1999.

69. Ibeneme SC, Ekanem C, Ezuma A, Iloanusi N, Lasebikan N, Lasebikan O, Oboh O. Walking balance is mediated by muscle strength and bone mineral density in postmenopausal women: an observational study. BMC musculoskeletal disorders. 2018;19(1):1-10

70. Jordan AN, Jurca GM, Locke CT, Church TS, Blair SN. Pedometer indices for weekly physical activity recommendations in postmenopausal women. Medicine and science in sports and exercise. 2005;37(9):1627-32

71. Bryant CX, Green DJ. ACE personal trainer manual: The ultimate resource for fitness professionals: Recording for the Blind \& Dyslexic; 2006.

72. Abernathy R, Black D. Healthy body weights: an alternative perspective. The American journal of clinical nutrition. 1996;63(3):448S-51S

73. Bolzán AD, Bianchi MS, Bianchi NO. Superoxide dismutase, catalase and glutathione peroxidase activities in human blood: influence of sex, age and cigarette smoking. Clinical biochemistry. 1997;30(6):449-54

74. Gerard C, Brown KA. Obesity and breast cancer-Role of estrogens and the molecular underpinnings of aromatase regulation in breast adipose tissue. Molecular and cellular endocrinology. 2018;466:1530 
75. Lorincz A, Sukumar S. Molecular links between obesity and breast cancer. Endocrine-related cancer. 2006;13(2):279-92

76. Ponticelli D, D'Ambrosio A, Mastrogiacomo FR, Agozzino E. Long-term survivors of breast cancer diagnosis: a descriptive study on risk factors, characteristics of the disease and on lifestyles of a cohort of women attending a territorial oncological service. Euromediterranean Biomedical Journal. 2019;14

77. Friedenreich CM, Courneya KS, Bryant HE. Case-control study of anthropometric measures and breast cancer risk. International journal of cancer. 2002;99(3):445-52

78. Gandhi A, Copson E, Eccles D, Durcan L, Howell A, Morris J, Howell S, McDiarmid S, Sellers K, Evans DG. Predictors of weight gain in a cohort of premenopausal early breast cancer patients receiving chemotherapy. The Breast. 2019;45:1-6

79. Klassen O, Schmidt ME, Ulrich CM, Schneeweiss A, Potthoff K, Steindorf K, Wiskemann J. Muscle strength in breast cancer patients receiving different treatment regimes. Journal of cachexia, sarcopenia and muscle. 2017;8(2):305-16

80. Tudor-Locke $C$. Taking steps toward increased physical activity: Using pedometers to measure and motivate. President's Council on Physical Fitness and Sports Research Digest. 2002

81. Kobayashi J, Murase Y, Asano A, Nohara A, Kawashiri M-a, Inazu A, Yamagishi M, Mabuchi H. Effect of walking with a pedometer on serum lipid and adiponectin levels in Japanese middle-aged men. Journal of atherosclerosis and thrombosis. 2006;13(4):197-201

82. Tudor-Locke C, Ainsworth BE, Thompson RW, Matthews CE. Comparison of pedometer and accelerometer measures of free-living physical activity. Medicine and science in sports and exercise. 2002;34(12):2045-51

83. Bailey BW, Borup P, Tucker L, LeCheminant J, Allen M, Hebbert W. Steps measured by pedometry and the relationship to adiposity in college women. Journal of Physical Activity and Health. 2014;11(6):1225-32

84. Goodwin PJ, Ennis M, Pritchard KI, Trudeau ME, Koo J, Madarnas Y, Hartwick W, Hoffman B, Hood N. Fasting insulin and outcome in early-stage breast cancer: results of a prospective cohort study. Journal of clinical oncology. 2002;20(1):42-51

85. Kroenke $\mathrm{CH}$, Chen WY, Rosner B, Holmes MD. Weight, weight gain, and survival after breast cancer diagnosis. Journal of clinical oncology. 2005;23(7):1370-8

86. Kumar NB, Riccardi D, Cantor A, Dalton K, Allen K. A Case-Control Study Evaluating the Association of Purposeful Physical Activity, Body Fat Distribution, and Steroid Hormones on Premenopausal Breast Cancer Risk. The breast journal. 2005;11(4):266-72

87. Connolly BS, Barnett C, Vogt KN, Li T, Stone J, Boyd NF. A meta-analysis of published literature on waist-to-hip ratio and risk of breast cancer. Nutrition and cancer. 2002;44(2):127-38

88. Chiu C-H, Ko M-C, Wu L-S, Yeh D-P, Kan N-W, Lee P-F, Hsieh J-W, Tseng C-Y, Ho C-C. Benefits of different intensity of aerobic exercise in modulating body composition among obese young adults: a pilot randomized controlled trial. Health and quality of life outcomes. 2017;15(1):1-9 
89. Wewege M, Van Den Berg R, Ward R, Keech A. The effects of high-intensity interval training vs. moderate-intensity continuous training on body composition in overweight and obese adults: a systematic review and meta-analysis. Obesity Reviews. 2017;18(6):635-46

90. Winningham M, MacVicar M, Bondoc M, Anderson J, Minton J, editors. Effect of aerobic exercise on body weight and composition in patients with breast cancer on adjuvant chemotherapy. Oncology nursing forum; 1989.

91. Winningham ML, MacVicar MG, editors. The effect of aerobic exercise on patient reports of nausea. Oncology nursing forum; 1988.

92. Lazar MA. How obesity causes diabetes: not a tall tale. Science. 2005;307(5708):373-5

93. Ouchi N, Parker JL, Lugus JJ, Walsh K. Adipokines in inflammation and metabolic disease. Nature reviews immunology. 2011;11(2):85-97

94. Ojala BE, Page LA, Moore MA, Thompson LV. Effects of inactivity on glycolytic capacity of single skeletal muscle fibers in adult and aged rats. Biological research for nursing. 2001;3(2):88-95

95. Shima E, Hino M, Yamane T, Aoyama Y, Nakamae H, Yamamura R, Makita K, Sugano Y, Yasuda S, Takubo T. Acute rhabdomyolysis following administration of high-dose cyclophosphamide: case report. Annals of hematology. 2002;81(1):55-6

96. Tabata N, Tanaka R, Suga S, Mitani Y, Nakano T, Ido M, Azuma E, Ito M, Hamazaki M, Shiraishi T. Rhabdomyolysis following administration of cyclophosphamide: a case report in a BMT recipient. Bone marrow transplantation. 1996;17(6):1167-9

97. Becic T, Studenik C, Hoffmann G. Exercise increases adiponectin and reduces leptin levels in prediabetic and diabetic individuals: systematic review and meta-analysis of randomized controlled trials. Medical sciences. 2018;6(4):97

98. Shalamzari SA, Daneshfar A, Sablouei MH, Singh MAF, Kazemi A. The Effect of Aerobic Training on Tumor Growth, Adiponectin, Leptin and Ghrelin in Mice Models of Breast Cancer. Iranian Red Crescent Medical Journal. 2018;20(2)

99. Sirico F, Bianco A, D'Alicandro G, Castaldo C, Montagnani S, Spera R, Di Meglio F, Nurzynska D. Effects of physical exercise on adiponectin, leptin, and inflammatory markers in childhood obesity: systematic review and meta-analysis. Childhood obesity. 2018;14(4):207-17

100. Travier N, Buckland G, Vendrell JJ, Fernandez-Veledo S, Peiró I, Del Barco S, Pernas S, Zamora E, Bellet $\mathrm{M}$, Margeli M. Changes in metabolic risk, insulin resistance, leptin and adiponectin following a lifestyle intervention in overweight and obese breast cancer survivors. European journal of cancer care. 2018;27(4):e12861

101. Tremblay A, Dutheil F, Drapeau V, Metz L, Lesour B, Chapier R, Pereira B, Verney J, Baker JS, Vinet A. Long-term effects of high-intensity resistance and endurance exercise on plasma leptin and ghrelin in overweight individuals: The RESOLVE Study. Applied Physiology, Nutrition, and Metabolism. 2019;44(11):1172-9

102. Lynch BM, Nguyen NH, Moore MM, Reeves MM, Rosenberg DE, Boyle T, Vallance JK, Milton S, Friedenreich CM, English DR. A randomized controlled trial of a wearable technology-based 
intervention for increasing moderate to vigorous physical activity and reducing sedentary behavior in breast cancer survivors: The ACTIVATE Trial. Cancer. 2019;125(16):2846-55

103. Carioca A, Verde S, Luzia L, Rondó P, Latorre M, Ellery T, Damasceno N. Association of oxidative stress biomarkers with adiposity and clinical staging in women with breast cancer. European journal of clinical nutrition. 2015;69(11):1256-61

\section{Plate}

Plate 1 is available in the Supplemental Files section.

\section{Figures}

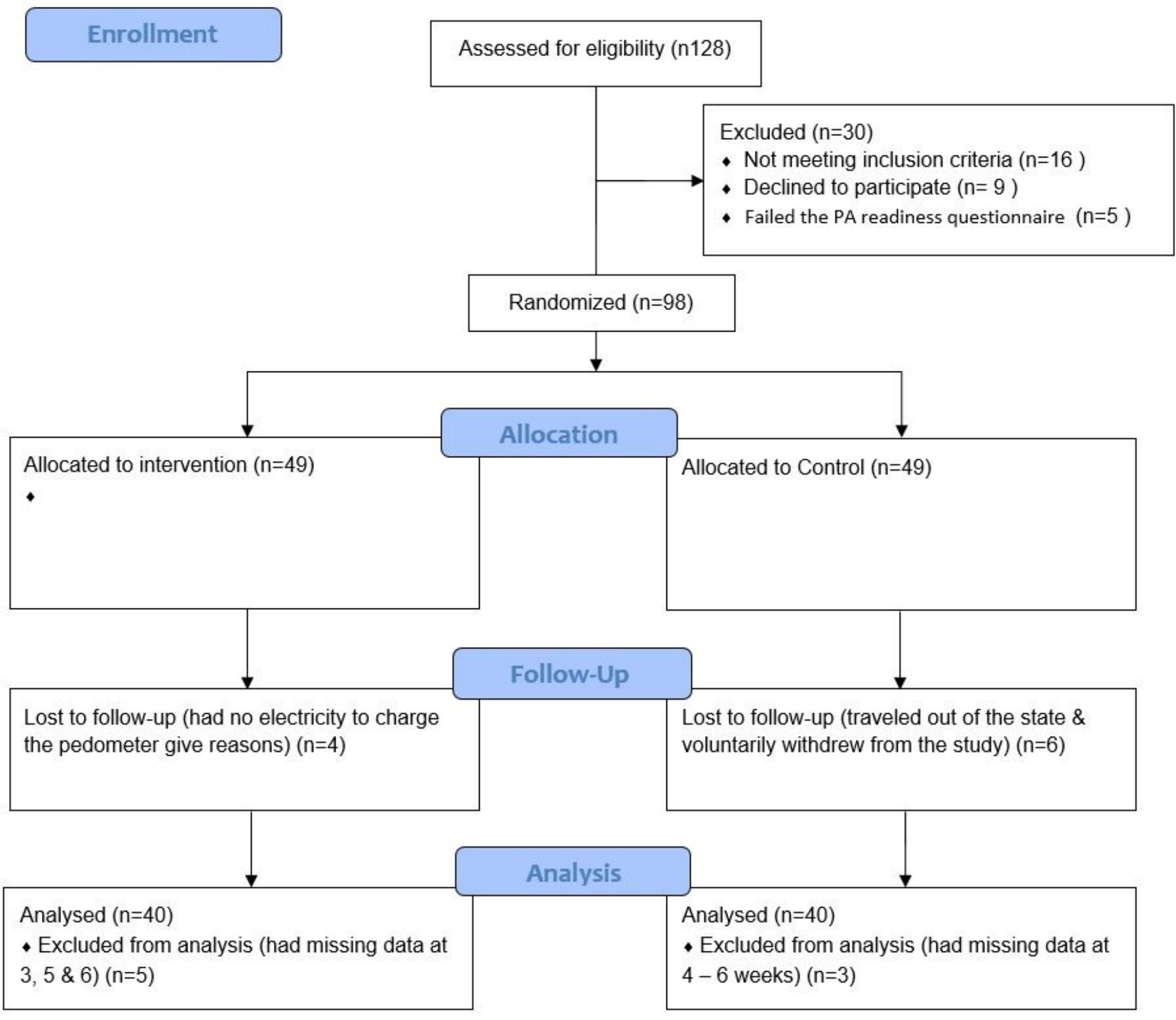


Figure 1

Design and flow of participants through the study

\section{Supplementary Files}

This is a list of supplementary files associated with this preprint. Click to download.

- Plate1.docx 